\title{
Aplicativo móvel para o cuidado da úlcera do pé diabético
}

\author{
Mobile application for the management of diabetic foot ulcers \\ Aplicación móvil para el cuidado de úlceras de pie diabético
}

Rafael Colodetti ${ }^{1}$ io https://orcid.org//0000-0001-7642-3702

Thiago Nascimento do Prado ${ }^{1}$ if https://orcid.org/0000-0001-8132-6288

Maria Edla de Oliveira Bringuente ${ }^{1}$ io https://orcid.org/0000-0002-5151-5368

Sheilla Diniz Silveira Bicudo ${ }^{1}$ io https://orcid.org/0000-0002-0634-2015

Como citar:

Colodetti R, Prado TM, Bringuente ME, Bicudo SD. Aplicativo móvel para o cuidado da úlcera do pé diabético. Acta Paul Enferm. 2021;34:eAPE00702.

DOI

http://dx.doi.org/10.37689/actaape/2021A000702

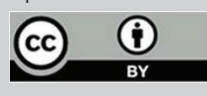

Descritores

Pé diabético; Aplicativos móveis; Informática em enfermagem; Informática médica; Software

Keywords

Diabetic foot; Mobile applications; Nursing Informatics; Medical informatics; Software

Descriptores

Pie diabético; Aplicaciones móviles; Informática Aplicada a la enfermería; Informática médica

Submetido

8 de Abril de 2020

Aceito

8 de Junho de 2020

\section{Autor correspondente}

Rafael Colodetti

E-mail: rcolodetti@hotmail.com

\section{Resumo}

Objetivo: Desenvolver e validar um aplicativo para dispositivos móveis que auxilie enfermeiros no processo de tomada de decisão do tratamento tópico na úlcera do pé diabético.

Métodos: Estudo metodológico realizado em quatro etapas: Definição de requisitos e elaboração do mapa conceitual do aplicativo; Geração das alternativas de implementação e prototipagem; Testes; Implementação. Para desenvolvimento do aplicativo utilizou-se software Nativescript para plataforma Android. Testes de usabilidade com 18 participantes, considerando as heurísticas de Nielsen e adaptações das heurísticas de usabilidade para dispositivos móveis.

Resultados: 0 aplicativo oferece informações atuais sobre a lesão do pé diabético como conceito, avaliação da úlcera, princípios do tratamento, recomendações clínicas para melhor cicatrização e os curativos para 0 cuidado tópico.

Conclusão: Os métodos estabelecidos para 0 desenvolvimento e validação revelaram-se adequados para atingir os objetivos propostos. Com a oferta dessa ferramenta tecnológica espera-se contribuir para o melhor cuidado da úlcera do pé diabético, contribuindo para uma prática de enfermagem baseada em evidência.

\section{Abstract}

Objective: To develop and validate a mobile application that help nurses in the decision-making process with regard to topical treatment in diabetic foot ulcers.

Method: Methodological study carried out in four stages: Definition of requirements and construction of the conceptual map of the application; Generation of alternatives for implementation and prototyping; Tests; Implementation. The software Nativescript for Android was used for the development of the application. Usability tests were carried out with 18 participants, considering Nielsen's heuristics and adaptations of usability heuristics for mobile devices.

Results: The application provides current information about the concept of diabetic foot ulcer, ulcer assessment, principles of treatment, clinical recommendations for better healing and dressings for topical care.

Conclusion: The methods used for development and validation proved to be adequate to achieve the proposed objectives. The implementation of this technological tool is expected to contribute to a better management of diabetic foot ulcers, contributing to an evidence-based nursing practice.

\section{Resumen}

Objetivo: Desarrollar y validar una aplicación para dispositivos móviles que ayude a enfermeros en el proceso de toma de decisiones del tratamiento tópico de úlceras de pie diabético. 
Métodos: Estudio metodológico realizado en cuatro etapas: definición de requisitos y elaboración del mapa conceptual de la aplicación; generación de alternativas de implementación y prototipo; pruebas; implementación. Para el desarrollo de la aplicación se utilizó el software Nativescript para plataforma Android. Pruebas de usabilidad con 18 participantes, considerando las heurísticas de Nielsen y adaptaciones de las heurísticas de usabilidad para dispositivos móviles.

Resultados: La aplicación ofrece información actual sobre úlceras de pie diabético, como concepto, evaluación de las úlceras, principios del tratamiento, recomendaciones clínicas para una mejor cicatrización y vendajes para el cuidado tópico.

Conclusión: Los métodos establecidos para el desarrollo y validación demostraron ser adecuados para alcanzar los objetivos propuestos. Con la oferta de esta herramienta tecnológica se espera contribuir para un mejor cuidado de úlceras de pie diabético y para una práctica de enfermería basada en evidencia.

\section{Introdução}

O quadro epidemiológico do Diabetes mellitus (DM) é preocupante, visto que, mundialmente, uma em cada onze pessoas tem essa síndrome metabólica (cerca de 463 milhóes dos adultos de 20 a 79 anos), sendo que a cada oito segundos, um indivíduo morre devido ao diabetes (aproximadamente quatro milhóes de mortes) e, em 2045, estima-se que, um adulto em dez (700 milhóes) terão DM. O Brasil é o terceiro país com maior número de crianças e adolescentes com DM tipo 1 e o quinto com maior soma de sujeitos com DM (16,8 milhôes).. ${ }^{(1)}$

Ressalta-se que o aumento da prevalência de DM é propício para trazer um aumento concomitante de suas complicaçóes. Sendo as mais comuns, e que atingem $50 \%$ das pessoas acima de 60 anos, a neuropatia periférica e o pé diabético $(\mathrm{PD}) .^{(2-4)}$

A neuropatia periférica é caracterizada por uma série de síndromes clínicas que acometem o sistema nervoso periférico sensitivo, motor e autonômico, e tem como principal desfecho, as úlceras nos pés associadas às isquemias, deformidades e/ou infecçóes, o denominado PD. ${ }^{(5)}$ Essa combinação é comum e responsável por um aumento substancial das taxas de morbimortalidade nos indivíduos com DM..$^{(6,7)}$

Estima-se que um membro inferior é perdido a cada 20 segundos, por causa das úlceras do pé diabético (UPD), não só afetando seriamente a qualidade de vida do paciente, mas também, aumentando o risco de amputação contralateral em 50 a 60\% no período de três a cinco anos..$^{(8-10)}$

Logo, o processo de decisão envolvido no manuseio da UPD visa à cicatrização primária sem evolução para amputação com o objetivo de conseguir o melhor desfecho possível para o paciente, a cicatrização da úlcera no menor tempo possível, o que nem sempre é atingido. ${ }^{(11,12)}$ Uma intervenção bem conduzida e no momento adequado é crucial para evitar a evolução para a UPD e amputação. ${ }^{(13)}$

Neste contexto, os enfermeiros possuem atuação fundamental na facilitação e na utilização da Prática Baseada em Evidências (PBE), de forma a se tornarem responsáveis pelo atendimento e orientação aos pacientes. ${ }^{(14)} \mathrm{A} \mathrm{PBE}$ baseia-se na integração da melhor evidência de pesquisa com valores de experiência clínica para facilitar a tomada de decisão, podendo ainda, tornar-se vital na melhoria da assistência de enfermagem. ${ }^{(15-17)}$

Ao longo dos últimos quinze anos, a expectativa de que a PBE fosse aplicada na prática da informática em saúde, ganhou destaque. Esse conceito é chamado de informática de saúde baseada em evidências, sendo definido como o uso consciencioso, explícito e judicioso das melhores evidências atuais ao tomar decisóes sobre a introdução e operação de tecnologias em informática (TI) em determinado ambiente de saúde. ${ }^{(18)}$

A utilização de aplicativo (app) móvel no contexto educacional vem crescendo, abrindo espaço para o mobile learning, que envolve o uso do dispositivo móvel, sozinho ou em combinação com outras TI, para promover o aprendizado. ${ }^{(19)}$ Os dispositivos móveis, principalmente os smartphones, apresentam novas oportunidades e desafios no campo das TI e na sociedade, como acesso ubíquo, portabilidade, democratização do acesso à informação, oportunidades de interaçáo e complexidade reduzida. ${ }^{(20)}$

$\mathrm{Na}$ enfermagem é crescente o desenvolvimento e utilizaçáo das TI, configurando-se em um mecanismo didático contemporâneo, que privilegia a automatização de processos, servindo inclusive para aumentar a segurança no processo decisório do cuidado. ${ }^{(21)}$ Nesta perspectiva, alguns estudos foram desenvolvidos em um ambiente virtual de aprendizagem com o intuito de oferecer meios para facili- 
tar o aprendizado da execução de atividades como avaliação e tratamento de feridas, gerenciamento e enfermagem psiquiátrica. ${ }^{(22-25)}$

Portanto, torna-se essencial implementar cuidados sistematizados baseados em evidências, sendo a TI uma ferramenta capaz de proporcionar a articulação entre teoria, prática e pesquisa. Nesse sentido, este estudo tem por objetivo desenvolver e validar app para dispositivos móveis que auxilie enfermeiros no processo de tomada de decisão do tratamento tópico na UPD.

\section{Métodos}

Trata-se de um estudo metodológico aprovado pelo Comitê de Ética em Pesquisa do Centro de Ciências da Saúde da Universidade Federal do Espírito Santo, (UFES) sob CAAE no 66019217.5.0000.5060. Em consonância com a Resolução no 466/2012 do Conselho Nacional de Saúde cumpriu-se as exigências para a proteçáo dos participantes de pesquisas científicas que envolvem seres humanos. Os sujeitos foram informados sobre o objetivo do estudo, esclarecidos com relação à assinatura do Termo de Consentimento Livre e Esclarecido.

Todas as etapas do estudo foram desenvolvidas em parceria entre o Programa de Pós-Graduação em Enfermagem e o Laboratório e Observatório de Ontologias Projetuais (LOOP), vinculado ao departamento de Desenho Industrial, Centro de Artes, UFES. Além disso, a norma ABNT ISO/ TR 16982:2014, intitulada ergonomia da interação humano-sistema - métodos de usabilidade que apoiam o projeto centrado no usuário subsidiou o desenvolvimento do app. A organização deu-se em ciclos de design iterativos que compreendem açóes de ensino, pesquisa e extensão vinculadas ao LOOP.

$\mathrm{O}$ app denominado UPDAPP foi desenvolvido utilizando o sistema operacional Android por ser uma plataforma distribuída gratuita e amplamente pela Google Play. As imagens utilizadas foram extraídas de uma publicação, após devida autorização do autor. ${ }^{(26)}$

O estudo foi realizado em quatro etapas: definição de requisitos e elaboração do mapa conceitual do app; geração das alternativas de implementação e prototipagem; testes; e implementação.

$\mathrm{Na}$ primeira etapa definiu-se que a base teórica seria fundamentada por publicaçóes sobre o tratamento da UPD, baseadas em evidências científicas, disponíveis em 2015 e revisitadas em 2020, na Cochrane Database of Systematic Reviews, Internacional Working Group Diabetic Foot e National Institute for Health and Care Excellence, objetivando definir os requisitos e elaboração do mapa conceitual (MC) do aplicativo. A revisão da literatura intrumentalizou a produção textual sobre o cuidado tópico da UPD. A partir dessa construção elaborou-se o conteúdo de cada tela e dos tutoriais.

Para que a equipe responsável pudesse iniciar o desenvolvimento do protótipo criou-se uma matriz organizacional. Essa ferramenta possibilitou a confecção de uma apresentação gráfica que organiza e representa o conhecimento, o MC (Figura 1). ${ }^{(27)}$

$\mathrm{Na}$ segunda etapa, o MC possibilitou à equipe do LOOP desenvolver novos passos para organização do processo em ciclos de iteração e gerar alternativas, a partir da utilização do software Nativescript para as funcionalidades do app, tendo em vista a adoção de tecnologias livres e abertas, sempre que possível. Protótipos funcionais (alpha, beta e pré-lançamento) das funcionalidades foram integrados à estrutura geral do UPDAPP, a fim de realizar testes reais com os usuários em potencial, a enfermagem.

Para desenvolvimento da terceira etapa, observou-se a norma ABNT ISO/IEC 25062:2011 que recomenda amostragem mínima de oito participantes. ${ }^{(28)} \mathrm{O}$ estudo contou no total com 18 partícipes, sendo oito avaliadores, da equipe do LOOP, constituindo o Grupo G1; e dez enfermeiros assistenciais, atuantes na atenção primária e secundária, com mínimo de dois anos de experiência, e diretamente ligados ao cuidado com o PD, compondo o Grupo G2. Todos os avaliadores foram selecionados de forma aleatória. As avaliaçôes ocorreram no mês de outubro de 2017.

Os testes ocorreram em dois momentos. No primeiro momento, o app foi inspecionado pelo G1, através de uma técnica realizada por um conjunto de princípios e diretrizes, as heurísticas. Uma forma de caracterizar a usabilidade dos sistemas é a avaliação 


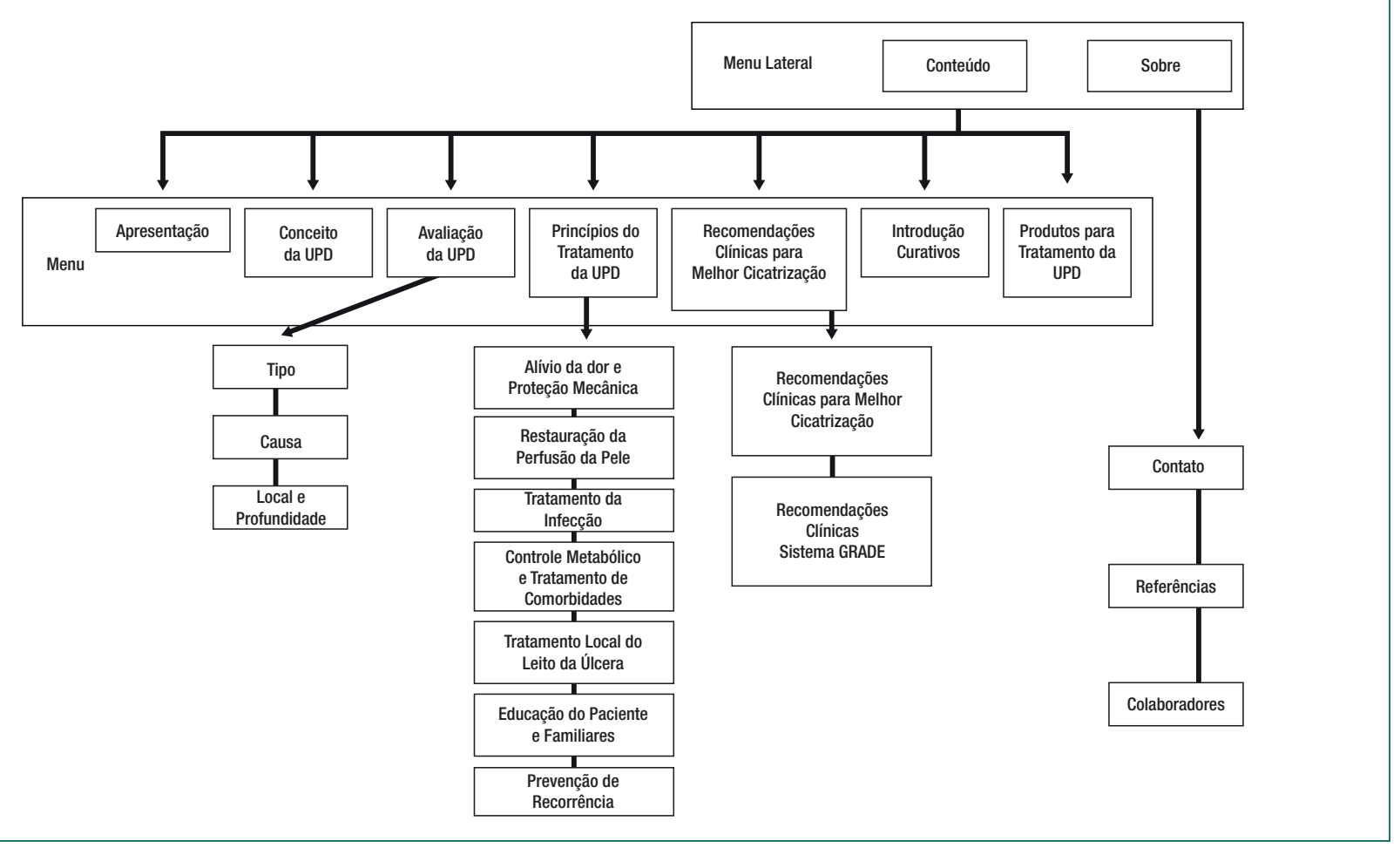

Figura 1. Mapa Conceitual do aplicativo UPDAPP

heurística (AH), sendo: 1) Visibilidade do sistema; 2) Correspondência entre o sistema e o mundo real; 3) Controle e liberdade do usuário; 4) Consistência e padronização; 5) Reconhecimento em vez de memorização; 6) Flexibilidade e eficiência de uso; 7) Projeto estético e minimalista; 8) Prevenção de erros; 9) Auxílio aos usuários a reconhecer, diagnosticar e se recuperar de erros; 10) Ajuda e documentação. ${ }^{(29)}$

Mais três heurísticas específicas, adaptadas e validadas, de usabilidade para celulares com touchscreen foram incluídas: 1) Pouca interação homem/dispositivo; 2) Interação física e ergonomia; 3) Legibilidade e layout. Visando proporcionar ao usuário uma melhor interação e tornando a interface mais amigável. ${ }^{(30)}$

Unificando as heurísticas encontradas foi proposto um conjunto de princípios e diretrizes customizadas, resultando em um checklist para compor essa AH. ${ }^{(31)}$ Logo, os avaliadores receberam esse instrumento para inspecionar todo o sistema com o objetivo de identificar possíveis erros na interface, baseando-se nos princípios do design.

Assim, cada problema era registrado, sendo informado o número de avaliadores que o identifi- caram, o local onde o mesmo ocorreu e, também, sua classificação por um grau de severidade, que ainda, foi dividida em: 1) Problema Cosmético; 2) Problema Pequeno; 3) Problema Grande; 4) Problema Catastrófico. Também foram coletados dados referentes a heurística violada, assim como, sugestōes para possíveis correçôes. ${ }^{(31)}$

No segundo momento, visando encontrar problemas de usabilidade enfrentados pelo público-alvo, o app foi avaliado e validado pelo Grupo G2, individualmente. Foram selecionadas dez enfermeiras, com idade entre 28 a 50 anos, com experiência no tratamento da UPD e no manuseio de smartphones.

A avaliaçáo ocorreu em dois passos, sendo que no primeiro distribuiu-se um roteiro com cinco questóes sobre o cuidado tópico que deveriam ser respondidas buscando-se suporte no conteúdo disponibilizado pelo app, por conseguinte todo o processo de manuseio e reaçóes do usuário foi observado. Assim como, o período de tempo, em minutos, que cada profissional precisou para efetuar as tarefas.

O segundo passo transcorreu através do preenchimento de questionário, validado e adaptado. $\mathrm{O}$ 
mesmo utilizou a escala de Likert que permite respostas entre um (discordo fortemente) a cinco (concordo fortemente); a pontuação três foi indicada em casos de dúvida se concorda ou discorda, ou caso, o avaliador náo se sentisse apto para responder; as pontuaçóes iguais ou superiores a quatro foram consideradas como adequadas. ${ }^{32,33)}$

Estando o aplicativo em perfeitas condiçôes para inserção na internet, após as devidas correçóes, foi executado a quarta etapa, a implementação. Consistiu-se na disponibilização do UPDAPP no provedor da UFES e na loja virtual do Google Play.

\section{Resultados}

As telas elaboradas seguiram a seguinte sequência: informaçóes gerais sobre o app, colaboradores do seu desenvolvimento, referências utilizadas e contato do autor. A tela inicial é a Home com as seguintes opçóes de acesso: Apresentação, Conceito da UPD, Avaliação da UPD, Princípios do Tratamento, Recomendações Clínicas e Produtos para Tratamento, Referências, Colaboradores e Contato com o Autor.

Ao clicar na opção Apresentação, o usuário terá acesso às informaçôes gerais do app. Acessando a opção Conceito da UPD revela-se a definição do termo com imagem ilustrativa. Em Avaliação da UPD, o enfermeiro é orientado a seguir uma estratégia padronizada e consistente para avaliar a ferida, com algumas opçóes de acesso, a fim de classificá-la quanto ao Tipo, Causa, Local e Profundidade e a presença de Sinais de Infecção. Já em Princípios do Tratamento são disponibilizados para consulta, os sete princípios do tratamento da UPD, que contribuem para a cicatrização da maioria das lesóes (Figura 2).

Continuando, a tela Recomendaçôes Clínicas para melhor cicatrização disponibiliza cada recomendação formulada com base no sistema padronizado de classificação GRADE (Grading of Recommendations Assessment, Development and Evaluation), tanto a qualidade da evidência (classificada em quatro níveis que representam a confiança: alto, moderado, baixo, muito baixo), quanto da força da recomendação da evidência (expressa a ênfase para que seja adotada ou rejeitada uma determinada conduta em forte ou fraca). ${ }^{(34)}$

Na sequência, temos Produtos para Tratamento, com três opções de acesso: Introdução Curativos, Curativo Ideal e Opçóes de Curativos. Na tela Introduçáo Curativos destaca-se que as coberturas são uma parte fundamental do cuidado. Em Curativo Ideal são descritas as características de um penso ideal e as opçóes de coberturas indicadas. Prosseguindo, as Opçóes de Curativos, ilustram os agentes tópicos recomendados para o tratamento da lesão.

A avaliação de usabilidade realizada pelo G1 resultou na detecção de oito problemas de severidade grande e dois pequenos. Foram considerados erros grandes: usuário sempre ser forçado a regressar ao menu somente com o botão voltar, podendo este

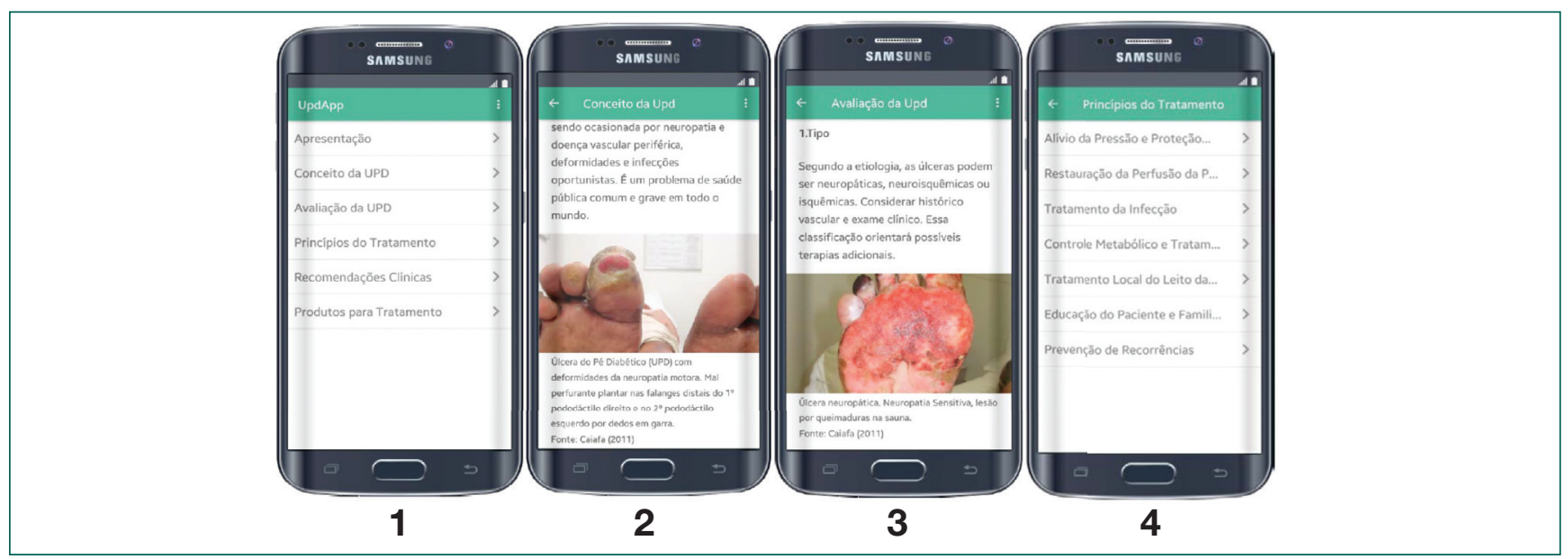

Figura 2. 1) Home do app; 2) Conceito da UPD; 3) Avaliação da UPD (tipo); 4) Princípios do tratamento 
se cansar por repetir constantemente essa ação (seis avaliadores); telas de conteúdo não apresentarem componentes de navegaçáo, não sendo informado o número de páginas, seçóes percorridas ou telas restantes para consulta (quatro avaliadores); seçáo Produtos para Tratamento não possuía nome adequado, visto que, seu conteúdo aborda técnicas e opçóes de tratamento, e não somente, de opçóes de curativos (um avaliador); os títulos serem extensos, não cabendo na tela, possibilitando certa dificuldade ao usuário saber ao certo, do que se trata o item (três avaliadores); não oferecer mecanismos para uma navegação dinâmica, ir de um tela para outra com apenas um click (três avaliadores); ser exigido do usuário usar a barra de rolagem (scroll) nas telas de conteúdo (um avaliador); em Opçóes de Curativos, o título ainda se mantém como Produtos para Tratamento (um avaliador); e todos os submenus direcionarem para o início da mesma página (dois avaliadores). Ponderando-se os problemas de severidade pequena, um avaliador considerou que o botão de voltar não informava para onde o usuário era direcionado e três avaliadores sugeriram mudança do título Para uma Melhor Cicatrização, a fim de melhor entendimento.

$\mathrm{Na}$ avaliação e validação realizada pelo $\mathrm{G} 2$, na primeira etapa, nenhum usuário ultrapassou o tempo de dez minutos. No total foram cometidos três erros na execução das atividades propostas, todos relacionados à dificuldade em encontrar as respostas para as questóes. Por exemplo: o usuário acessava o item do menu Princípios do Tratamento, na tentativa de responder a atividade sobre o sistema GRADE, sendo que a consulta deveria ser realizada no item Recomendações Clínicas. Na segunda etapa, a partir das respostas obtidas dos questionários, obteve-se a média e o desvio padrão, conforme demonstrado na tabela 1 . Ressalta-se que um participante não foi considerado por não ter respondido dois itens.

Quando calculada a média das respostas dos usuários G2, as pontuaçóes variaram entre 4,2 a 4,9. Desta forma, o app foi considerado adequado em todos os quesitos avaliados. Uma segunda análise foi executada por meio das respostas de usuários diferentes para a mesma pergunta. Essas médias va-
Tabela 1. Média e desvio padrão das respostas da enfermagem

\begin{tabular}{l|cc}
\hline Perguntas & $\begin{array}{c}\text { Média das } \\
\text { respostas }\end{array}$ & $\begin{array}{c}\text { Desvio } \\
\text { padrão }\end{array}$ \\
\hline $\begin{array}{l}\text { 1. Você tem facilidade no uso de aplicativos móveis } \\
\text { (funcionalidade) }\end{array}$ & 4,5 & 0,70 \\
$\begin{array}{l}\text { 2. } 0 \text { software é preciso na execução de suas funções } \\
\text { (funcionalidade) }\end{array}$ & 4,7 & 0,67 \\
$\begin{array}{l}\text { 3. O software dispõe das principais funções necessárias para } \\
\text { tratar a úlcera do pé diabético (funcionalidade) }\end{array}$ & 4,4 & 1,26 \\
$\begin{array}{l}\text { 4. O software reage adequadamente quando ocorrem falhas } \\
\text { (confiabilidade) }\end{array}$ & 4 & 1,05 \\
$\begin{array}{l}\text { 5. É fácil entender o conceito e aplicação do software } \\
\text { (usabilidade) }\end{array}$ & 5 & 0 \\
6. É fácil aprender a usar 0 software (usabilidade) & 5 & 0 \\
$\begin{array}{l}\text { 7. O software oferece ajuda de forma clara (usabilidade) } \\
\text { 8. O tutorial do software é de fácil entendimento (usabilidade) } \\
\text { 9. O tempo de execução do software é adequado (eficiência) }\end{array}$ & 4,5 & 0,52 \\
$\begin{array}{l}\text { 10. Os recursos disponibilizados no software são adequados. } \\
\text { (eficiência) }\end{array}$ & 4,9 & 0,31 \\
\hline
\end{tabular}

riaram entre 4 a 5. Com o propósito de identificar a variabilidade das respostas da enfermagem em torno da média, calculou-se o desvio padrão (DP). No menor DP foi encontrado 0,31 (sem convergência) e no maior 1,26 (para maiores divergências).

Quando questionados se o app reagia adequadamente na ocorrência de falha, as respostas do G1 retrataram uma diversificação maior do que o G2. Nas perguntas 5 a 10, identificou-se uma variabilidade menor, revelando que para manuseio do aplicativo, não houve dificuldade.

\section{Discussão}

A Avaliação Heurística e os testes realizados, com os avaliadores (G1) e com os usuários (G2), respectivamente, forneceram informaçôes importantes sobre a usabilidade e validação do UPDAPP, sendo demonstrado que o app é fácil de usar, compreender o seu conceito e aplicação, proporcionando ajuda de forma clara.

Contudo, foi sugerido a criação de um padrão de navegação estruturado para proporcionar um meio de localização (breadcumbs); melhora da mobilidade das janelas utilizadas no sistema com implementação de barras de rolagem ancoradas, a fim de permitir que o texto contínuo, imagens ou qualquer outro elemento, pudessem ser "rolados", além do fechamento de links; e padronização das fontes das figuras. 
Sendo a usabilidade um atributo principal da qualidade de qualquer produto interativo, em especial, nos aparelhos touchscreen, a mesma deve ser considerada quando no lançamento de um produto, podendo assim, ser um diferencial do UPDAPP em um mercado de pressa, como é o dessas tecnologias.

Como a AH é baseada no conhecimento e na experiência de especialistas avaliadores de softwares, que analisando as interfaces de um determinado sistema fazem o levantamento dos possíveis problemas e sugerem soluçóes, o método empregado demonstrou ser simples, com duração inferior a duas horas e de menor custo. Ressalta-se, também, a importância da participação dos usuários. Por isso, foi priorizada a participação de profissionais de enfermagem.

Comprovou-se a precisão na execução e oferta das funções necessárias para tratar a UPD (funcionalidade), reação adequada em caso de falhas (confiabilidade) e tempo de execução adequado (eficiência). Que da mesma forma são perfis contemplados na avaliação da qualidade de um app que privilegia a visão do usuário. Outros aspectos relevantes para construção de materiais digitais são a organização, interpretaçáo e visualizaçáo, de modo que estes sejam atrativos e favoreçam a reflexão e a reelaboração do conhecimento. Para tanto, a quantidade de informação inserida em cada tela e sua apresentação devem ser precisas, simples e diretas. Logo, itens como tamanho e tipo de fonte, escolha das cores e os contrastes entre elas foram cuidadosamente selecionados.

Nenhum usuário ultrapassou o tempo de dez minutos, sendo isso um ponto positivo, pois este não deve exceder esse período no manuseio de um app em teste. ${ }^{(35)}$

Nesse escopo, pode-se ressaltar a importância do desenvolvimento dessa ferramenta em um contexto multidisciplinar, possibilitando assim, o interesse de outros profissionais para a criação de tecnologias para a área da saúde com a integraçáo de conhecimentos. No cenário da educação superior em enfermagem, a tecnologia proporcionará inúmeras oportunidades, podendo ser empregada no processo de ensino-aprendizagem pelo estudante com a finalidade de construir o próprio conhecimento, bem como auxiliar o educador na formação interativa para o ensino, sendo uma alternativa para a fomentação do pensar e criar.

No que se refere à tecnologia móvel utilizada, em geral, os usuários não apresentaram dificuldades, pois se familiarizam com esse tipo de dispositivo e os obstáculos pontuados foram minimizados ou corrigidos. De maneira geral, o app UPDAPP traz uma contribuição importante para a enfermagem, desmitificando informaçôes sobre condutas, dispondo dos recursos necessários para auxiliar na tomada de decisão sobre o cuidado tópico da UPD, sobretudo, aumentando o seu conhecimento científico e reverberando de forma positiva na sua prática profissional.

Entre as limitações identificadas destacam-se que as heurísticas possuem questóes muito gerais para dispositivos móveis, algumas delas não sendo aplicadas ao UPDAPP. Além disso, o app foi desenvolvido apenas na plataforma Android. Outra limitação consistiu na impossibilidade de aumentar o tamanho das imagens apresentadas nas telas.

\section{Conclusão}

Os objetivos delineados para esse estudo foram alcançados, principalmente quanto ao desenvolvimento e validação do app proposto. Diante da realidade do uso das tecnologias no ensino, especialmente na enfermagem, percebe-se que a ferramenta para subsídio a tomada de decisão sobre o cuidado tópico da UPD, em tecnologia móvel, é uma proposta didática, interativa e imprescindível para implementação e disseminação da prática baseada em evidências. Quando o cuidado é prestado tendo como eixo norteador essa abordagem, além de intensificar o julgamento clínico, favorece a integração do conhecimento, pesquisa e prática pela qualificação e incorporação de uma assistência mais distinta e efetiva, fundamentada na ciência.

\section{Agradecimentos}

Os autores agradecem o Prof. Dr. Hugo Cristo Sant' Anna e ao Laboratório Observatório de 
Ontologias Projetuais (LOOP) do curso de Design da Universidade Federal do Espírito Santo (UFES), em especial Isabel Zaneti Zucarato, Rafael Batista Magalhães, Rodrigo Bins Gomes, Fabrício Broedel Silva Nunes, Rhamily Queiroz Lima, Tarsila Aragáo Costa de Oliveira e Julio César Reis Alves. Aos colegas enfermeiros que participaram da avaliação e validação do aplicativo. Desejamos estender nossos agradecimentos a Profa. Dra. Paula Cristina de Andrade Pires Olympio (em memória) pelas contribuiçóes e análise crítica do artigo.

\section{Colaborações}

\section{Colodetti R, Prado TN, Bringuente MEO e Bicudo} SDS declaram que contribuíram com a concepção projeto, análise e interpretação dos dados, redaçáo do artigo, revisão crítica relevante do conteúdo intelectual e aprovação da versão final a ser publicada.

\section{Referências}

1. International Diabetes Federation (IDF). Diabetes atlas. 9th ed. Bruxelas: IDF; 2019.

2. Pop-Busui R, Boulton AJM, Feldman EL, Bril V, Freeman R, Malik RA, Sosenko JM, Ziegler D. Neuropatia diabética: posicionamento da Associação Americana de Diabetes. Diabetes Care. 2017; 40:136-54.

3. Brasil. Ministério da Saúde. Secretaria de Atenção à Saúde. Estratégias para o cuidado da pessoa com doença crônica: diabetes mellitus. Brasília (DF): Ministério da Saúde; 2016.

4. Nascimento OJ, Pupe CC, Cavalcanti EB. Neuropatia diabética. Rev Dor. 2016;17 Suppl 1:S46-51.

5. International Working Group on the Diabetic Foot (IWGDF). International consensus on the diabetic foot and practical guidelines on the management and the prevention of the diabetic foot. [Internet]. IEGDF; 2015 [cited 2019 Dec 5]. Available from: http://iwgdf.org/guidelines

6. Noor S, Khan RU, Ahmad J. Understanding diabetic foot infection and its management. Diabetes Metab Syndr. 2017;11(2):149-56.

7. American Diabetes Association. Microvascular complications and foot care: standards of medical care in diabetes 2019. Diabetes Care. 2019;42 Suppl 1:S124-38.

8. Santos KP,LuzSC,Mochizuki L, d'OrsiE. Carga da doença paraas amputações de membros inferiores atribuíveis ao diabetes mellitus no Estado de Santa Catarina, Brasil, 2008-2013. Cad Saude Publica. 2018;34(1):e00013116.

9. Li X, Xu G, Chen J. Tissue engineered skin for diabetic foot ulcers: a meta-analysis. Int J Clin Exp Med. 2015;8(10):18191-6.

10. Sociedade Brasileira de Diabetes. Diretrizes da Sociedade Brasileira de Diabetes 2019-2020. São Paulo: Clannad; 2020.
11. Bakker K, Apelqvist J, Schaper NC. Practical guidelines on the management and prevention of the diabetic foot. Diabetes Metab Res Rev. 2012; 28 Suppl 1:225-31.

12. Jesscoate WJ. Wound healing - a practical algorihm. Diabetes Metabolism Research and Reviews. 2012; 28 Suppl 1:85-8.

13. Pedrosa HC, Tavares FS. As vias para ulceração. In: Pedrosa HC, Vilar L, Boulton AJ, editors. Neuropatias e pé diabético (cap.10). Rio de Janeiro: AC Farmacêutica; 2014.

14. Camargo FC, Iwamoto HH, Monteiro DAT, Lorena LT. Avaliação de intervenção para difusão da enfermagem baseada em evidências em hospital de ensino. Rev Gaúcha Enferm. 2016; 37(esp):e68962:1-9.

15. Schneider LR, Pereira RP, Ferraz L. A prática baseada em evidência no contexto da Atenção Primária à Saúde. Saúde Debate. 2018;42(118):594-605.

16. Camargo FC, Iwamoto HH, Galvão CM, Pereira GA, Andrade RB, Masso GC. Competences and barriers for the evidence-based practice in nursing: an integrative review [Internet]. Rev Bras Enferm. 2018;71(4):2030-8.

17. Pedrosa KK, Oliveira IC, Feijão AR, Machado RC. Enfermagem baseada em evidência: caracterização dos estudos no Brasil. Cogitare Enferm. 2015;20(4):733-41.

18. Rigby M, Ammenwerth E, Beuscart-Zephir MC, Brender J, Hyppönen $H$, Melia S, et al. Evidence Based Health Informatics: 10 years of efforts to promote the principle. Yearb Med Inform. 2013;8(1):34-46.

19. Mather C, Cummings $E$, Gale F. Mobile learning in nursing: tales from the profession. Stud Health Technol Inform. 2018;252:112-7.

20. Feijó VC, Gonçalves BS, Gomez LS. Heurística para avaliação de usabilidade em interfaces de aplicativos smartphones: utilidade, produtividade e imersão. Design \& Tecnologia. 2013; 3(6):33-42.

21. Frias MA. Vivência de graduandos de enfermagem no uso do ambiente virtual de aprendizagem [tese]. São Paulo (SP): Escola de Enfermagem, Universidade de São Paulo; 2015.

22. Pereira MC, Melo MR, Silva AS, Évora YD. Evaluation of a webquest on the theme "management of material resources in nursing" by undergraduate students. Rev Lat Am Enfermagem. 2010;18(6):1107-14.

23. Camacho AC, Tenório DM, Silva RP, Barreto BM, Oliveira BG. Evaluation of the interactive blog on wound repair and nursing care. Rev Pesqui Cuid Fundam. 2013;5(3):202-10.

24. Holanda VR, Pinheiro AK. Desenvolvimento de um sistema hipermídia para 0 ensino interativo das doenças sexualmente transmissíveis. Rev Enferm UFPE. 2015; 9 Supl:2:781-9.

25. Oliveira RM, Duarte AF, Alves D, Furegato AR. Desenvolvimento do aplicativo Tabaco Quest para informatização de coleta de dados sobre tabagismo na enfermagem psiquiátrica. Rev Lat Am Enfermagem. 2016;24 e2726.

26. Caiafa JS, Castro AA, Fidelis C, Santos VP, Silva ES, Sitrângulo C Jr. Atenção integral ao portador de pé diabético. J Vasc Bras. 2011;10(4):1-32.

27. Novak JD. Aprender criar e utilizar o conhecimento. Mapas conceituais como ferramentas de facilitação nas escolas e empresas = Learning, creating and using knowledge. Lisboa: Plátano Editora; 2000.

28. Associação Brasileira de Normas Técnicas (ABNT). NBR ISO/EC 25062:2011. Engenharia de Softwere - Requisitos e avaliação de qualidade de produto de software (SQuaRe) - Formato comum da Indústria (FCl) para relatórios de teste de usabilidade [Internet]. Brasília (DF); ABNT; 2011.

29. Nielsen J. Heuristic evaluation. In: Nielsen J, Mack RL, editors. Usability inspection methods, Heuristic Evaluation. New York: John Wiley \& Sons, Inc.; 1994. 
30. Krone C. Validação de heurísticas de usabilidade para celulares touchscreen. Grupo de Qualidade de Software (GQS). Florianópolis (SC): Universidade Federal de Santa Catarina; 2013.

31. Salazar LA, Lacerda TC, Wangenheim CG, Barbalho RA. Customizando Heurísticas de Usabilidade para Celulares. Cuiabá (MT): Simpósio Brasileiro de Fatores Humanos em Sistemas Computacionais; 2012.

32. Tibes CM, Dias JD, Zem-Mascarenhas SH. Aplicativos móveis desenvolvidos para a área da saúde no Brasil: Revisão Integrativa da Literatura. Rev Min Enferm. 2014;18(2):471-8.
33. Vescovi SB, Bicudo SD, Primo CC, Sant'anna HC, Bringuente ME, Rohr RV. Aplicativo móvel para avaliação dos pés de pessoas com diabetes mellitus [dissertação]. Vitória: Universidade Federal do Espírito Santo; 2017.

34. Morche J, Conrad S, Passon A, Perleth M, Gartlehner G, Meerpohl JJ, et al. [GRADE Guidelines: 16. GRADE evidence to decision frameworks for tests in clinical practice and public health]. Z Evid Fortbild Qual Gesundhwes. 2018;133:58-66. German.

35. Rogers Y, Sharp H. Preece J. Design de interação. Porto Alegre: Bookman Editora; 2013. 